\title{
Atypical Fracture of the Sternum After Long-Term Alendronate Plus Cholecalciferol Treatment: A Case Report
}

\author{
Luis H. Martín Arias ${ }^{1}$ - Pilar García Ortega ${ }^{2}$ María Sáinz Gil ${ }^{1}$ (D) \\ Ester Navarro García ${ }^{3}$ Carlos Treceño Lobato ${ }^{3}$ - Virginia Delgado Armas $^{3}$
}

Published online: 28 February 2017

(C) The Author(s) 2017. This article is published with open access at Springerlink.com

\begin{abstract}
A 55-year-old woman developed an atraumatic sternum fracture during treatment with alendronate for osteoporosis. The woman received alendronate $70 \mathrm{mg}$ in combination with cholecalciferol 5600 IU once weekly, as well as nonsteroidal anti-inflammatory drugs. After 4 years of treatment, following a dorsal flexion with no direct thoracic trauma, the patient suffered a fracture of the sternum, with an X-ray revealing sternal body fracture. This fracture was seen to be transverse, noncomminuted and without displacement. Magnetic resonance imaging was carried out to rule out the presence of either a pathological fracture or a fracture resulting from osteoporotic fragility, and showed a triple sternal fracture involving the body, as well as the upper and lower manubrium of the sternum. This fracture presented the features of an atypical femur fracture, except for the location. The alendronate and cholecalciferol combination was discontinued and denosumab was prescribed. After the withdrawal of alendronate, the patient showed clinical improvement, with a decrease in pain, and is currently having routine checkups. The causality algorithm of the Spanish Pharmacovigilance System shows a score of 5, indicating a possible
\end{abstract}

MaríaSáinz Gil

maria.sainz@uva.es

1 Facultad de Medicina, Centro de Farmacovigilancia de Castilla y León, Centro de Estudios sobre la Seguridad de Medicamentos (CESME), C/Ramón y Cajal, $n^{\circ} 7$, Universidad de Valladolid, C.P. 47005 Valladolid, Spain

2 Centro de Salud Sur - P. Alameda - Covaresa, Salud Castilla y León, Ctra. De Rueda, 137, C.P. 47008 Valladolid, Spain

3 Facultad de Medicina, Centro de Estudios sobre la Seguridad de Medicamentos (CESME), C/Ramón y Cajal, $n^{\circ} 7$, Universidad de Valladolid, 47005 Valladolid, Spain relationship between the patient's sternum fracture and her use of the suspect drug (Naranjo scale $6=$ probable).

\section{Key Points}

Long-term treatment with bisphosphonates could induce atypical fractures in anatomical locations other than the femur.

Bisphosphonate treatment should be reconsidered in patients after long-term use.

All suspected adverse drug reactions must be reported to the pharmacovigilance systems in order to detect potential alarm signals related to drug use.

\section{Introduction}

Bisphosphonates are synthetic drugs that bind to bone hydroxyapatite crystals and inhibit the action of osteoclasts, thus preventing both in vitro and in vivo bone resorption. Approximately 50\% of the drug is deposited in the bone; however, the time it stays in the bone or the potential long-term effects are not accurately known.

Prolonged bisphosphonate therapy has been linked to atypical fractures. The risk seems to be related to the suppression of bone turnover, which, in turn, may favour certain types of fractures [1]. Thus far, the fractures that have been described as bisphosphonate therapy-related atypical fractures share a certain number of clinical and radiological features, i.e. they are likely to be located at the subtrochanteric area of the femur; a previous trauma cannot 
be found or trauma is minimal; they are noncomminuted, transverse or slightly oblique; and they may be either complete or incomplete fractures [2]. To our knowledge, this is the first report of a sternum fracture that was likely to be related to bisphosphonate therapy. In addition, it is the first case that occurred with no trauma and involving a nonelderly female patient that has been reported to the Spanish Pharmacovigilance System.

\section{Case Report}

The patient was a 55-year-old female with a history of smoking since the age of 16 years (20 cigarettes/day), with no other cardiovascular risk factors and presenting with a body mass index (BMI) of $25 \mathrm{~kg} / \mathrm{m}^{2}$. She reached menopause at the age of 53 years. The patient had no other concomitant conditions, and her medical record indicated that she had not been previously treated with corticoids.

In 2010, following an intense lumbar effort, the patient experienced severe lumbar pain. Lumbar X-ray and computed tomography (CT) revealed D11 fracture and L1 partial collapse. Femoral and lumbar dual energy X-ray absorptiometry (DEXA) densitometry showed a total femur bone density of $0.770 \mathrm{~g} / \mathrm{cm}^{2}(T$-score $1.9 ; Z$-score 1.6) and a bone mineral density on lumbar spine of $0.881 \mathrm{~g} / \mathrm{cm}^{2}$ ( $T$-score $2.5 ; Z$-score 1.8$)$, consistent with femoral osteopenia and lumbar osteoporosis, according to the World Health Organization (WHO) criteria. Accordingly, in September 2010, the patient was prescribed a combination of alendronate $70 \mathrm{mg}$ and cholecalciferol 5600 IU once weekly, as well as nonsteroidal anti-inflammatory drugs. Improvement was noted and since then periodical routine densitometry tests have been carried out, which showed femoral and lumbar osteopenia (Table 1). In October 2013, the patient continued to complain of chronic dorsolumbar pain.

In April 2014, the patient felt a sensation of stabbing pain in the sternal area while sitting up in bed, followed by pain while moving and breathing, but no dyspnoea. She had not suffered any external force (i.e. pushing) or trauma.
Because of these alarming symptoms, she came to the hospital Emergency Department. Physical examination disclosed a swollen, painful area located on the sternal region of the body. Coronary involvement was ruled out. Sternal X-ray revealed sternal body fracture, which was seen to be transverse, noncomminuted and without displacement. Magnetic resonance imaging (MRI) was undertaken to rule out the presence of either a pathological fracture or a fracture resulting from osteoporotic fragility. MRI showed a triple sternal fracture involving the body, as well as the upper and lower manubrium of the sternum (Fig. 1). Pathological fracture was ruled out; however, an old pathological fracture of the eleventh dorsal vertebra (D11) with several vertebral collapses at D4, D7 and D8 was noted.

Metabolic study to dismiss potential secondary osteoporosis-related causes and tests aimed at ruling out other bone diseases (i.e. hyperparathyroidism, hypothyroidism, bone tumours or metastases, calcium-phosphorus metabolism disorders, and vitamin D deficiency) was performed. All these tests yielded normal results, except for vitamin D [25 ng/L (range 30-80)]. This slight hypovitaminosis D does not seem to be clinically relevant, and is thus insufficient to induce this kind of fracture, involving three parts of the sternum. The results of densitometry carried out in May 2014 were consistent with total femur osteopenia and lumbar spine osteopenia (Table 1); therefore, other factors that could have contributed to the development of this fracture were ruled out. Due to suspicion of an alendronaterelated fracture, the alendronate plus cholecalciferol combination was discontinued and the specialist who followed this patient decided to prescribe denosumab. The patient was treated with calcium and vitamin D during the entire process.

After the withdrawal of alendronate, the patient showed clinical improvement, with a decrease in pain, and is currently having routine checkups.

The causality algorithm of the Spanish Pharmacovigilance System [3] shows a score of 5, indicating a possible relationship between the patient's sternum fracture and her use of the suspect drug (Naranjo scale 6, probable).

Table 1 Densitometry results performed on the patient and the resulting diagnosis (from 2010 to 2014)

\begin{tabular}{|c|c|c|c|c|c|c|c|c|}
\hline \multirow[t]{2}{*}{ Year } & \multicolumn{4}{|l|}{ Femoral densitometry } & \multicolumn{4}{|l|}{ Lumbar densitometry } \\
\hline & Bone mass density $\left(\mathrm{g} / \mathrm{cm}^{2}\right)$ & $T$-score & $Z$-score & Diagnosis & Bone mass density $\left(\mathrm{g} / \mathrm{cm}^{2}\right)$ & $T$-score & $Z$-score & Diagnosis \\
\hline 2010 & 0.770 & 1.9 & 1.6 & Osteopenia & 0.881 & 2.5 & 1.8 & Osteoporosis \\
\hline 2012 & 0.817 & 1.5 & 1.2 & Osteopenia & 0.955 & 1.9 & 1.1 & Osteopenia \\
\hline 2013 & 0.747 & 2.1 & 1.7 & Osteopenia & 0.939 & 2.0 & 1.1 & Osteopenia \\
\hline 2014 & 0.721 & 2.3 & 1.9 & Osteopenia & 0.920 & 2.2 & 1.3 & Osteopenia \\
\hline
\end{tabular}




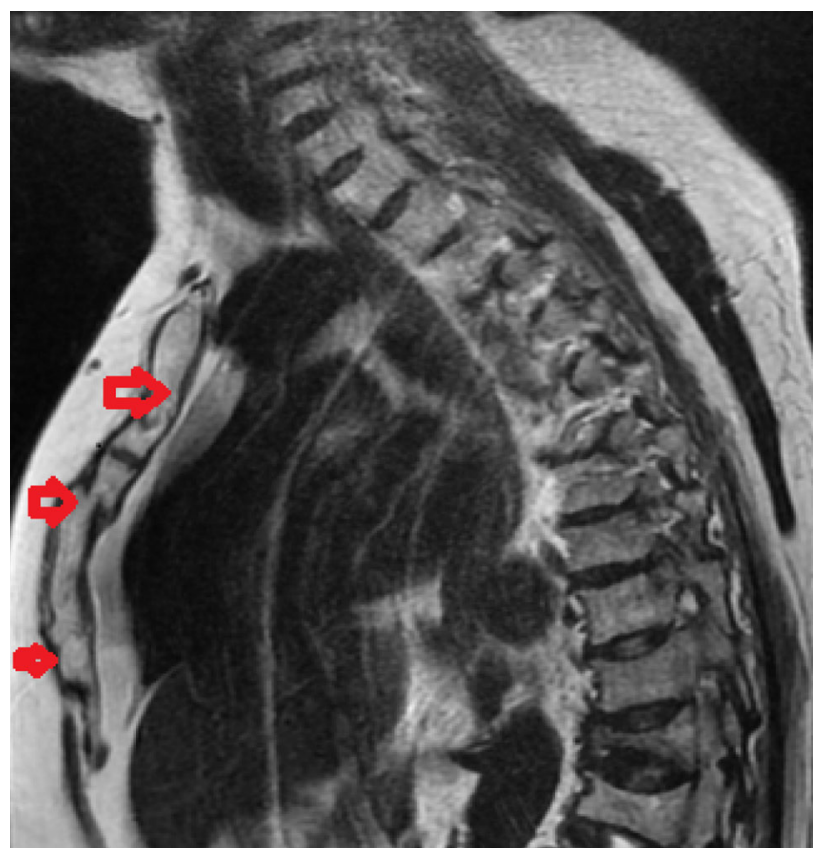

Fig. 1 Triple sternal fracture involving the body as well as the upper and lower manubrium of the sternum

\section{Discussion}

Sternal fractures are rare. They are most often caused by severe chest traumas that usually result from car accidents, sport lesions or violent attacks. In reviewing the literature, we found only three cases of patients with atraumatic fracture of the sternum. These patients presented wellestablished risk factors for this type of fracture and did not have a history of bisphosphonate therapy [4-6].

After a search of Medline, we failed to find any cases of bisphosphonate therapy-associated atypical sternal fractures; however, cases of this type of fracture in anatomical locations other than the sternum have been published.

We also conducted a search of the Spanish Pharmacovigilance System database (FEDRA, accessed July 2015), and encountered 121 suspected cases of bisphosphonate therapy-related fractures, with a total of 125 fractures, as, in some of these cases, more than one fracture was reported for a given patient. Most of these fractures involved the femur $(n=92)$, followed by fractures of the tibia $(n=4)$, jaw and spine $(n=3$ each), fibula $(n=2)$, and ribs, sternum, radius, hip, and ankle ( $n=1$ each), while anatomical location was not reported in 15 cases. The only fracture of the sternum in the FEDRA database corresponded to an 81-year-old female receiving bisphosphonates, which occurred because of a minimal trauma. It should be borne in mind that all of the above were just suspected cases because the cause-effect relationship with bisphosphonate therapy was not established in any of the cases. Nevertheless, the broad range of anatomical locations leads us to believe that the issue of atypical fractures may be more complex than originally thought since atypical fractures presumably involve locations other than the usual, particularly other than the femur.

In the present case, the causative role of the drug cannot be dismissed because the temporal sequence is suggestive and we were not able to find an alternative causal explanation. Thus, it should be noted that our patient's fracture occurred 4 years after the commencement of alendronate therapy. MRI findings led us to rule out a pathological fracture or a fracture caused by sternal osteoporosis, there was no severe direct sternal trauma (i.e. the most frequent cause for sternal fracture), the patient was only 55 years of age, she had not been previously treated with corticoids, and secondary bone diseases (i.e. metabolic, neoplastic or metastatic conditions) were ruled out, with the exception of the minor vitamin D deficiency, for which she was being treated. Furthermore, the features of our case suggest biological plausibility. Accordingly, we concluded that our patient's sternal fracture was likely to be related to her 4-year alendronate therapy.

Although resorption markers such as C-terminal telopeptide of type I collagen (CTX), N-terminal telopeptide (NTX), and deoxypyridinoline (DPD), which are useful for the follow-up of therapy compliance, were not measured, our patient's compliance was checked in her computerised medical record. Of note, all the drugs prescribed to the patient within the 4-year period of interest were stated in her medical record.

The fracture we report herein fulfilled the clinical and radiological features of the atypical femoral fractures, namely lack of previous trauma, and complete or incomplete transverse, noncomminuted fracture in a patient previously treated with bisphosphonates. The present fracture differs from the usual atypical fractures related to bisphosphonates in its location only.

\section{Conclusions}

Our patient had been treated with bisphosphonates for 4 years. Accordingly, we feel that this is a case of atypical fracture of the sternum that has not been previously reported in the literature, and which fulfils the well-known criteria for the atypical fracture of the femur. It is therefore our belief that the issue of atypical fractures is far more complex than originally thought since our case shows that these fractures can involve anatomical locations other than the usual. Thus, we recommend considering the potential diagnosis of an atypical fracture, even if it is not located on the femur, in all patients taking bisphosphonates who present with a new fracture. The case has been reported to the Spanish Pharmacovigilance System. 


\section{Compliance with Ethical Standards}

Informed consent Written informed consent was obtained from the patient for publication of this case report and any accompanying images. A copy of the written consent may be requested for review from the corresponding author.

Conflicts of interest Luis H. Martín Arias, Pilar García Ortega, María Sáinz Gil, Esther Navarro García, Carlos Treceño Lobato, and Virginia Delgado Armas declare that they have no conflicts of interest.

Funding No financial support was received for the conduct of this case report or the preparation of this article.

Open Access This article is distributed under the terms of the Creative Commons Attribution-NonCommercial 4.0 International License (http://creativecommons.org/licenses/by-nc/4.0/), which permits any noncommercial use, distribution, and reproduction in any medium, provided you give appropriate credit to the original author(s) and the source, provide a link to the Creative Commons license, and indicate if changes were made.

\section{References}

1. Park-Wyllie LY, Mamdani MM, Juurlink DN, et al. Bisphosphonate use and the risk of subtrochanteric or femoral shaft fractures in older women. JAMA. 2011;305:783-9.

2. Kwek EB, Goh SK, Koh JS, Png MA, Howe TS. An emerging pattern of subtrochanteric stress fractures: a longterm complication of alendronate therapy? Injury. 2008;39:224-31.

3. Aguirre C, García M. Causality assessment in reports on adverse drug reactions. Algorithm of Spanish pharmacovigilance system [in Spanish]. Med Clin (Barc). 2016;147:461-4.

4. Lin KH, Ponampalam R. Sternum insufficiency fracture presenting as acute chest pain: a case report and review of the literature. Eur J Emerg Med. 2006;13(2):122-4.

5. Horikawa A, Miyakoshi N, Kodama H, Shimada Y. Insufficiency fracture of the sternum simulating myocardial infarction: case report and review of the literature. Tohoku J Exp Med. 2007;211 (1):89-93.

6. Abrahamsen $\mathrm{S} \varnothing$, Madsen CF. Atraumatic sternum fracture. BMJ Case Rep. 2014;2014. doi:10.1136/bcr-2014-206683. 